Giornale

di Tecniche

Nefrologiche

\& Dialitiche

Anno X n 3

(C) Wichtig Editore 1998

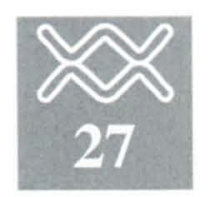

\title{
L'iperparatiroidismo secondario: linee guida per il trattamento ed effetti a lungo termine della terapia con calcitriolo endovena
}

\author{
M. Farina, F. Malberti, B. Corradi, P. Cosci, S. Mandolfo, E. Imbasciati \\ Servizio Dialisi - Ospedale Maggiore Lodi
}

\section{Fisiopatologia dell'iperparatiroidismo secondario}

ei pazienti con insufficienza renale cronica si può osservare una iperplasia delle ghiandole paratiroidee ed un conseguente aumento del paratormone (PTH) circolante già a partire da un filtrato glomerulare inferiore ad $80 \mathrm{~mL} / \mathrm{min}$.

Il PTH è uno dei principali fattori che intervengono nella regolazione del metabolismo minerale e di quello osseo. L'ormone è un polipeptide a singola catena di 84 aminoacidi la cui sintesi avviene nelle cellule paratiroidee in forma di precursore (preproPTH). Mentre è ancora in via di formazione esso penetra nel reticolo endoplasmatico dove prosegue la maturazione fino a formare il proPTH. Successivamente il pro-PTH perde una sequenza di 6 residui aminoacidici e viene immagazzinato nella sua forma definitiva (PTH 1-84) all'interno dei granuli secretori. Al momento della secrezione i granuli si fondono con la membrana cellulare e l'ormone entra in circolo. La sua azione negli organi bersaglio è mediata attraverso il legame con un recettore specifico (cui si lega anche il Parathyroid hormone-related peptide) recentemente clonato ed appartenente alla famiglia dei recettori accoppiati alle proteine $G$ (1). Questo recettore è espresso prevalentemente negli osteoblasti, nei condrociti e nelle cellule epiteliali renali (2). Il legame del PTH con il recettore attiva due messaggeri intracellulari, la adenilato ciclasi e la fosfolipasi $\mathrm{C}$ che, attraverso l'aumento del cAMP e dell'inositolo trifosfato rispettivamente, agiscono a cascata su altri segnali intracellulari con l'effetto di modulare numerose attività biologi- che (trascrizione genica, aumento del calcio intracellulare, ecc.) (2). Sul versante osseo l'eccesso di PTH provoca un'intensa attività di rimodellamento che consiste in aumento del riassorbimento osseo per reclutamento degli osteoclasti, attivazione degli osteoblasti (tuttavia non sufficiente a compensare l'eccessivo riassorbimento con conseguente perdita netta di massa ossea), eccessiva formazione di osso immaturo con aspetto "intrecciato" (woven), aumento della sintesi e deposizione di connettivo midollare (3-6). I meccanismi che concorrono all'eccessiva secrezione di PTH (7-9) sono riassunti nella Tabella I e verranno qui di seguito analizzati.

\section{TABELLA I - MECCANISMI RESPONSABILI DELL'ECCESSIVA SECREZIO- NE DI PTH}

\footnotetext{
- Ridotta sintesi e concentrazione plasmatica di calcitriolo

- Ritenzione dei fosfati inorganici

- Ipocalcemia ed alterata relazione $\mathrm{Ca} / \mathrm{PTH}$ (set point)

- Resistenza scheletrica al PH

Anomalie anatomo-funzionali delle ghiandole paratiroidee
} 


\section{1) Ridotta sintesi}

\section{e concentrazione plasmatica} di calcitriolo $\left(1,25(\mathrm{OH})_{2} \mathrm{D}_{3}\right)$

La forma metabolicamente attiva di questo ormone si forma in gran parte nelle cellule tubulari prossimali del rene, ma sono stati descritti anche siti di minore importanza sul piano clinico come la placenta, $i$ cheratociti ed i macrofagi (7). La riduzione della massa nefronica rappresenta il meccanismo fondamentale della diminuita sintesi di calcitriolo per ridotta idrossilazione in posizione $1 \alpha$ della 25 OHVit $\mathrm{D}_{3}$, precursore inattivo di derivazione epatica. Il deficit di calcitriolo provoca una netta diminuzione dell'assorbimento intestinale di calcio con tendenza all'ipocalcemia mentre, a livello osseo, è responsabile di una riduzione della matrice proteica di provenienza osteoblastica (osteocalcina, osteopontina) e della resistenza scheletrica all'azione del PTH con l'effetto di aggravare l'ipocalcemia e di produrre un ulteriore stimolo alla secrezione di PTH (8).

Sulle cellule paratiroidee il calcitriolo agisce attraverso un recettore specifico, prevalentemente nucleare e di tipo steroideo, il Vitamin D receptor (VDR) costituito da una singola catena polipeptidica di $50 \mathrm{kD}$. La frazione della 1,25-diidrossivitamina $D_{3}$ che si lega al VDR è quella libera, non legata alle proteine plasmatiche e quindi in grado di attraversare la membrana cellulare. Il legame del calcitriolo al VDR determina l'inibizione della sintesi del precursore del PTH (pre-pro PTH) attraverso una riduzione dell' attività trascrizionale del gene (9). La riduzione dei livelli di calcitriolo è inoltre in grado di indurre una proliferazione delle cellule paratiroidee sia mediante una down-regulation del VDR stesso (10) sia, come è stato recentemente osservato, attraverso la de-repressione di un fattore di crescita (il $c-m y c$ proto oncogeno) $(8,9,11-13)$.

\section{2) Ritenzione di fosfati}

La riduzione della massa nefronica è responsabile della ridotta escrezione urinaria di fosforo inorganico e della conseguente iperfosforemia. Secondo la classica trade-off hypothesis di Slatopolsky e Bricker (14) l'iperfosforemia è la causa primitiva della riduzione del calcio ionizzato plasmatico il quale, a sua volta, induce una ipersecrezione di PTH nel tentativo di riportare alla norma l'escrezione urinaria di fosfati e la calcemia. Secondo altri invece la causá prima dell'ipocalcemia è lo stesso deficit di vitamina $D$. L'iperfosforemia d'altra parte esercita una azione inibitoria diretta sulla sintesi di calcitriolo (inibizione della 1 $\alpha$-idrossilasi) mentre sulle cellule paratiroidee determina una maggior resitenza all'azione del calcitriolo, attiva la sintesi di PTH (studi in vitro) e stimola la secrezione con un meccanismo probabilmente post trascrizionale (7, 14-16). Sembra infine che la crescita delle stesse cellule paratiroidee sia inibita da una riduzione della fosforemia. Si pone quindi con evidenza come esista una complessa rete di fenomeni interdipendenti legata ai diversi fattori.

\section{3) Ipocalcemia}

L'ipocalcemia, comunque prodottasi, è un importante fattore di accellerazione dell'iperparatiroidismo secondario. Il calcio ionizzato (ossia la forma metabolicamente attiva del calcio) esplica la propria azione sulle cellule paratiroidee attraverso un recettore specifico, il calcium-sensing receptor (Ca-SR), recentemente clonato sul cromosoma 3q (17), lo stesso sul quale si trova il gene per il PTH. Il calcio ione interferisce con i meccanismi secretori del PTH mentre il calcitriolo agisce direttamente sulla via di sintesi. È nota da tempo una relazione inversa tra la concentrazione sierica di calcio ed i livelli plasmatici di PTH. La curva che esprime questa relazione ha forma sigmoidale ed in vivo si ottiene inducendo nel paziente ipo- ed iper-calcemia. La dinamica della secrezione paratiroidea può essere descritta attraverso alcuni parametri ricavati dall'analisi della curva calcio-PTH (18). Tra essi uno dei più importanti è il cosiddetto set-point della relazione calcio-PTH ovvero, secondo una delle definizioni, il livello di calcemia ionizzata cui corrisponde il 50\% della secrezione massima di PTH (19). È ancora discussa l'ipotesi di una alterazione diretta del set point nell'uremia. Per alcuni Autori vi è uno spostamento verso destra ed in alto della curva e pertanto la secrezione di PTH risulta aumentata anche con normali valori di calcemia; il dato non è tuttavia ammesso da altri $(8,18,20,21)$.

\section{4) Resistenza scheletrica all'azione calcemizzante del PTH}

La ridotta risposta calcemica all'azione del PTH è un'altra causa che può indurre lo sviluppo precoce di ipocalcemia in corso di insufficienza renale ed avviare la stimolazione delle ghiandole paratiroidee $(2,7,8,16$, 17). In altre parole si realizza una condizione in cui sono necessari livelli maggiori di PTH per mantenere normale la calcemia. I fattori responsabili di questo difetto sono soprattutto i ridotti livelli di calcitriolo, la ritenzione di fosfati ed una probabile downregulation dei recettori ossei per il PTH (Tab. II).

Studi sperimentali su animali e sull'uomo hanno dimostrato che la somministrazione di calcitriolo (22) e la restrizione dietetica di fosforo (23) correggono anche in modo indipendente la risposta calcemica al PTH. È
TABELLA II - CAUSE DI RESISTENZA SCHELETRICA AL PTH

- Carenza di calcitriolo

- Iperfosforemia

- Probabile down regulation del recettore specifico osseo per il paratormone (PTH/PTH-rP-R) 
probabile che la ritenzione dei fosfati agisca sia riducendo i livelli di calcitriolo sia interferendo con i meccanismi di mobilizzazione del calcio in sede ossea (24). È stato anche suggerito da altre osservazioni animali che aumentati livelli di PTH possono indurre una ridotta espressione sulla membrana cellulare dei recettori specifici per il PTH. Sembra tuttavia che tale fenomeno acquisti rilievo in presenza degli altri due fattori già citati (22).

\section{5) Anomalie anatomo-funzionali delle ghiandole paratiroidee}

La riduzione del numero dei VDRs e l'alterata sensibilità del Ca-SR sono la causa originaria dell'aumentata attività paratiroidea e costituiscono una spinta costante all' accrescimento della ghiandola. A questi fattori si aggiungono, secondo modalità non ancora ben definite, una serie di anomalie genomiche responsabili di un accrescimento anarchico con caratteri di monoclonalità (15). Il substrato istologico specifico che caratterizza l'evoluzione dell'iperparatiroidismo secondario verso la forma cosiddetta terzaria o refrattaria (al calcitriolo) è l'evoluzione da iperplasia diffusa a iperplasia nodulare $(16,25)$.

\section{Prevenzione}

La prevenzione delle conseguenze ossee dell'insufficienza renale (osteodistrofia uremica) è strettamente connessa al controllo dell'iperparatiroidismo secondario, alla conservazione di una normale mineralizzazione ossea e deve evitare l'accumulo di elementi traccia tossici per l'osso come ad esempio l'alluminio, contenuto in una grande quantità di preparati antiacidi e protettivi della barriera mucosa gastrica. È importante considerare questi obbiettivi già durante il followup del paziente con insufficienza renale. La dieta ipoproteica è un mezzo semplice ed efficace oltre che nel rallentare la progressione del danno renale anche nel ridurre l'apporto di fosfati ed eventualmente, nei casi di incompleta adesione alla prescrizione alimentare, potrà essere integrata dal- l'impiego a basse dosi di chelanti. Quando poi il filtrato glomerulare cade al di sotto dei $30 \mathrm{~mL} / \mathrm{min}$ è spesso opportuno associare una supplementazione con calcitriolo, anch'esso inizialmente a basse dosi ed anche in assenza di ipocalcemia.

Nel paziente uremico in dialisi è parimenti di fondamentale importanza cercare di ottenere la normalizzazione della calcemia e della fosforemia mediante la personalizzazione della composizione del liquido di dialisi, l'impiego farmacologico di metaboliti attivi della vitamina D e la limitazione o l'abolizione di elementi traccia potenzialmente tossici contenuti come abbiamo visto anche in alcuni farmaci (26-29). In tutti i casi infine si dovranno evitare o correggere con attenzione i disordini dell' equilibrio acido-base ricorrendo alla prescrizione di bicarbonato nell'eventualità di acidosi metabolica.

\section{Controllo dell'iperfosforemia}

Il controllo della fosforemia è uno degli obbiettivi cardine nella prevenzione e nel trattamento dell'iperparatiroidismo secondario. La fosforemia va quanto più possibile mantenuta entro il limite normale, vale a dire fra 4 e $5 \mathrm{mg} / \mathrm{dL}$. Nei pazienti in dialisi, sia peritoneale che extracorporea, è necessario insistere sulla restrizione dell'apporto dietetico di fosfati e nella maggioranza dei casi impiegare chelanti del fosforo. I chelanti del fosforo attualmente più usati sono il calcio carbonato $(\mathrm{CaCO} 3)$ e il calcio acetato. Come abbiamo già visto l'impiego cronico di farmaci contenenti gel di idrossido di alluminio andrebbe - per quanto possibile - evitato nel paziente uremico per il rischio spesso sottovalutato di tossicità legata al metallo. Per aumentarne l'efficacia $i$ chelanti vanno assunti durante il pasto ad un dosaggio adeguato all'introito fosforico alimentare medio. Generalmente sono sufficienti dosi giornaliere di 2-8 g di calcio carbonato o acetato. Il contenuto di calcio elemento nel carbonato e nell' acetato di calcio è diverso, rispettivamente il $40 \%$ e il $25 \%$ del peso globale. L'efficacia dei diversi chelanti è legata anche al $\mathrm{pH}$ dell'ambiente gastrico: il calcio carbonato richiede infatti un $\mathrm{pH}$ acido per disciogliersi in calcio ionizzato e poter esplicare la sua azione chelante sul fosforo a livello dell'intestino tenue. Il calcio acetato invece è effìcace entro più ampi ranges di $\mathrm{pH}$ ambiente. Ne deriva che quest'ultimo sarà preferibile nei pazienti trattati contemporaneamente con alcalinizzanti o con farmaci inibitori della secrezione acida gastrica (anti-H2, anti-pompa protonica, ecc.) che tendono ad aumentare il $\mathrm{pH}$ ambiente gastrico. Una frequente conseguenza dell'impiego di chelanti contenenti calcio nel paziente in dialisi è l'ipercalcemia con un effetto dose-dipendente. Questa tendenza può essere corretta riducendo la concentrazione di calcio nel liquido di dialisi. L'ipercalcemia è meno frequente con l'impiego di calcio acetato che tra l'altro, a parità di calcio elemento assorbito, ha dimostrato una capacità chelante circa doppia rispetto al calcio carbonato. Il calcio acetato rispetto al carbonato ha tuttavia l'inconveniente di essere causa di retrogusto sgradevole con intuibili conseguenze sulla compliance dei pazienti.

Un altro chelante efficace è il carbonato di magnesio. La comparsa di diarrea (in genere per dosi $>2 \mathrm{~g} / \mathrm{die}$ ) ne limita l'uso ad alte dosi in monoterapia mentre la tendenza all'ipermagnesiemia può richiedere la riduzione del $\mathrm{Mg}++$ nel liquido di dialisi $(0.25$ $\mathrm{mmol} / \mathrm{L}$ pari a $0.6 \mathrm{mg} / \mathrm{dL}$ ). Più spesso è utile come terapia di associazione con gli altri chelanti in modo da ovviare l'effetto ipercalcemico. Nel paziente in dialisi peritoneale, dovendo garantire un apporto proteico di 1.2$1.4 \mathrm{~g} / \mathrm{kg} /$ die, l'introito di fosfati può arrivare a circa $20 \mathrm{mg} / \mathrm{hg} /$ die. L'apporto fosforico può essere anche maggiore se è elevato il consumo di cibi ad alto contenuto fosforico (es. latte e derivati, frattaglie, uova). Tali alimenti andrebbero quindi limitati come pure quelli contenenti conservanti a base di polifosfati (es. insaccati) o le bevande ricche in fosfati (es. 
TABELLA III - DOSAGGIO TERAPEUTICO DEI METABOLITI DELLA VITAMINA D NEI PAZIENTI IN DIALISI

Somministrazione continua, orale:

Calcitriolo 0.25-1 $\mu \mathrm{g} / \mathrm{die}$

1 - $\alpha$-idrossicolecalciferolo 0.25 - $1 \mu \mathrm{g} /$ die

25-idrossicolecalciferolo o calcifediolo 25 -100 $\mu \mathrm{g} / \mathrm{die}$

Somministrazione intermittente, orale o endovenosa:

Calcitriolo 1-5 $\mu \mathrm{g} \times 2-3 /$ settimana

\section{TABELLA IV - LINEE GUIDA PER IL TRATTAMENTO DELL'IPERPARATI- ROIDISMO SECONDARIO NEI PAZIENTI IN DIALISI}

Mantenimento della fosforemia tra 4 e $5 \mathrm{mg} / \mathrm{dL}$ :

Restrizione dietetica di fosforo $(<1000 \mathrm{mg} / \mathrm{die})$

Uso di calcio carbonato o acetato

Dose dialitica adeguata

Correzione dell'ipocalcemia:

Se PTH $>200 \mathrm{pg} / \mathrm{mL}$ : calcitriolo per os a basse dosi

Se PTH $<100 \mathrm{pg} / \mathrm{mL}$ : aumento della concentrazione del calcio nel dialisato

Iperparatiroidismo moderato (PTH $200-700 \mathrm{pg} / \mathrm{mL})$ e calcemia normale $(9-10.5 \mathrm{mg} / \mathrm{dL}$ ) Boli di calcitriolo per os $\mathrm{o}$ e.v. (iniziando con $\mathrm{I} \mu \mathrm{g} \times 2 /$ settimana)

Iperparatiroidismo severo (PTH $>700 \mathrm{pg} / \mathrm{mL}$ ) e calcemia normale: Boli di calcitriolo e.v. (iniziando con 3-4 $\mu \mathrm{g} \mathrm{x} 2 /$ settimana)

cola e birra). Una moderata restrizione dietetica riduce l'introito giornaliero di fosforo a $800-1000 \mathrm{mg}$ ed in questo caso il contemporaneo impiego di chelanti rende possibile un adeguato controllo della fosforemia. La quota di fosforo alimentare assorbita dall'intestino è infatti pari a circa il $50-70 \%$ di quella ingerita e si riduce al $30 \%$ con l'uso di questi prodotti. Così, ad esempio, se l'apporto alimentare è di di $1000 \mathrm{mg}$ avremo un assorbimento intestinale ridotto a 300 $\mathrm{mg}$ che corrisponde approssimativamente alla quantità che viene eliminata con la procedura dialitica realizzandosi un bilancio netto in pareggio.

\section{Mantenimento di normali livelli di calcemia}

Il mantenimento della calcemia nel range di normalità è indispensabile per evitare la stimolazione delle ghiandole paratiroidee e per avere un' adeguata mineralizzazione ossea.

Un ruolo importante nell'omeostasi calcica del paziente in dialisi è giocato dalla concentrazione di calcio nel liquido di dialisi. La concentrazione standard di calcio nelle soluzioni per dialisi peritoneale è $7 \mathrm{mg} / \mathrm{dL}(1.75$ $\mathrm{mmol} / \mathrm{L}$ ). Con tale concentrazione si hanno bilanci peritoneali modestamente positivi (10-20 mg per scambio), che sono opportuni per i pazienti che tendono all'ipocalcemia o che utilizzano basse dosi di sali di calcio. Nei pazienti trattati con alte dosi di calcio carbonato o con calcitriolo o che hanno tendenza all'ipercalcemia è invece opportuno utilizzare una concentrazione di calcio nel dialisato di 5 $\mathrm{mg} / \mathrm{dL}$, che comporta bilanci peritoneali modestamente negativi (da -10 a $-20 \mathrm{mg}$ per scambio). In emodialisi con un dialisato contenente 1.75 $\mathrm{mmol} / \mathrm{L}$ di calcio si hanno bilanci po- sitivi e si ottiene un incremento della calcemia ionizzata durante la seduta. Impiegando un bagno contenente 1.5 $\mathrm{mmol} / \mathrm{L}$ di calcio i bilanci sono approssimativamente in pareggio e la calcemia ionizzata al termine della seduta è in genere ai limiti superiori della norma. Quando il contenuto di calcio nel dialisato scende a 1.25 $\mathrm{mmol} / \mathrm{L}$ si hanno bilanci negativi e la calcemia ionizzata a fine seduta scende ai limiti inferiori della norma (1.10-1.20 $\mathrm{mmol} / \mathrm{L})$ determinando spesso un aumento del PTH sierico rispetto ai livelli pre-dialitici. Il bilancio del calcio sia in dialisi peritoneale che in emodialisi è influenzato significativamente dalla calcemia basale e dalla ultrafiltrazione (bilancio convettivo). In emodialisi per ogni litro di acqua rimossa si ha una perdita di circa $60 \mathrm{mg}$ di calcio $(30,31)$.

\section{Livelli target di PTH}

È stato dimostrato che nel paziente uremico il turnover osseo è conservato solo quando i livelli di PTH sono 2-3 volte quelli normali. Al contrario se i livelli di PTH sono nel range di normalità il turnover è spesso ridotto e si può sviluppare una osteopatia adinamica. Diversi Autori suggeriscono quindi di mantenere i livelli di PTH tra 100 e 200 pg/mL (v.n. 10-65 $\mathrm{pg} / \mathrm{mL}$ ) sospendendo il calcitriolo se essi si riducono al di sotto dei 100 $\mathrm{pg} / \mathrm{mL}(32,33)$.

\section{Terapia dell'iperparatiroidismo secondario}

\section{Metaboliti della vitamina D}

Il controllo della calcemia è un importante target nella prevenzione e cura dell'iperparatiroidismo secondario al pari di quello della fosforemia. Numerosi pazienti tuttavia sviluppano un'iperparatiroidismo progressivo nonostante adeguati livelli di entrambi questi fattori. Studi recenti hanno documentato che la secrezione di 
PTH viene inibita in modo diretto dal calcitriolo (34). Visto che il deficit di calcitriolo ha un ruolo determinante nello sviluppo dell'iperparatiroidismo secondario molti pazienti devono essere trattati con metaboliti della vitamina D. Il criterio principale per iniziare il trattamento con calcitriolo nei pazienti in dialisi è la presenza di livelli di PTH > $200 \mathrm{pg} / \mathrm{mL}$ e probabilmente il cut-off scende a $100 \mathrm{pg} / \mathrm{mL}$ in pre-dialisi. Ormai è stata ampiamente documentata 1'efficacia di diversi metaboliti della vitamina $D$ che, impiegati a dosaggio farmacologico, consentono di prevenire l'insorgenza e migliorano i segni clinici, biochimici, radiologici e istologici dell'iperparatiroidismo. Tuttavia i derivati $1-\alpha$ idrossilati e in particolare il calcitriolo vanno preferiti per la loro emivita più breve e per l'effetto diretto di inibizione sulla secrezione del PTH. Nella Tabella III sono riportati i dosaggi dei metaboliti della vitamina D usati nei pazienti in dialisi.

\section{Criteri guida per il trattamento con calcitriolo}

Nella Tabella IV sono riportati i criteri guida per il trattamento dell'iperparatiroidismo.

Nei pazienti con iperparatiroidismo lieve o moderato (PTH 200-700 $\mathrm{pg} / \mathrm{mL}$ e calcemia ridotta o ai limiti inferiori) il controllo della calcemia e della fosforemia e l'impiego del calcitriolo sono in genere misure sufficienti ad ottenere un buon controllo dei livelli di PTH. Al contrario dosi anche molto elevate di calcitriolo possono risultare inefficaci nei pazienti con forme avanzate. Si tratta generalmente di pazienti in cui si è sviluppata una marcata iperplasia delle paratiroidi spesso associata, in proporzione variabile, a lesioni nodulari (iperplasia nodulare). Nelle ghiandole con iperplasia nodulare è stata dimostrata una ridotta densità dei recettori per la vitamina $\mathrm{D}$ che alcuni considerano la principale causa di resistenza alla terapia (35). Non si può tuttavia escludere che a questo titolo giochi

TABELLA V - MODIFICAZIONI DELLO SCHEMA TERAPEUTICO NEI PAZIENTI CON IPERPARATIROIDISMO SECONDARIO IN BASE AI DATI DI LABORATORIO

\section{ACCETTABILE}

PTH $100-200 \mathrm{pg} / \mathrm{mL}$ Calcemia 9-10.5 mg/dL Fosforemia $4-5.5 \mathrm{mg} / \mathrm{dL}$ Prodotto $\mathrm{Ca} \times \mathrm{P}<60 \mathrm{mg} / \mathrm{dL}$

Se PTH $<100 \mathrm{pg} / \mathrm{mL}$

- Ridurre o sosp. calcitriolo

Se PTH $>20 \mathrm{pg} / \mathrm{mL}$

- Ridurre il Ca nel dialisato (es. $1.25 \mathrm{mmol} / \mathrm{L}$ )

- Ca acetato e/o MgCO3

Normocalcemía

Ipercalcemia ( $\geq 12 \mathrm{~g} / \mathrm{dL}$ )

- Aumentare calcitriolo

In caso di iperfosforemia e normocalcemia

- Aumentare la dose di calcio carbonato o acetato

In caso di ipocalcemia e normofosforemia

- Aumentare il calcitriolo un ruolo anche il fenomeno della down-regulation recettoriale (10). Per questo motivo è opportuno trattare e controllare l'iperparatiroidismo precocemente mediante la` somministrazione regolare di calcitriolo prima che l'iperplasia paratiroidea sfugga verso quadri di autonomia funzionale e la secrezione di PTH non risulti piú sopprimibile.

Nei casi di iperparatiroidismo avanzato è opportuno iniziare direttamente con un trattamento intermittente ad alte dosi riducendo la concentrazione di calcio nella soluzione per dialisi peritoneale o in quella per emodialisi a $5 \mathrm{mg} / \mathrm{dL}$ così da abbassare il rischio di ipercalcemia. La maggior efficacia del trattamento intermittente con boli ad alte dosi rispetto a quello continuo (0.25-0.5 $\mu \mathrm{g} / \mathrm{die})$ sembra legato alla possibilità di ottenere picchi plasmatici più elevati con maggior saturazione dei recettori paratiroidei per il calcitriolo $(36,37)$. Anche se la cinetica del farmaco somministrato per via endovenosa genera picchi plasmatici ancora più elevati se confrontati con il bolo orale, studi recenti sono concordi nel sostenere che non esistono differenze nella risposta tra le due modalità di somministrazione (38). A nostro avviso è opportuno iniziare il trattamento con due somministrazioni settimanali di $1 \mu \mathrm{g}$ ciascuna. La dose verrà quindi modulata in relazione ai dati di calcemia e fosforemia.

Per i pazienti con forme severe di iperparatiroidismo $(\mathrm{PTH}>700$ $\mathrm{pg} / \mathrm{mL}$ ) proponiamo un trattatamento più aggressivo iniziando con alte dosi di calcitriolo e.v. (Tab. V).

Nel 50-70\% dei soggetti con importante iperparatiroidismo secondario trattati secondo queste modalità si osservano: 1) una progressiva caduta dei livelli PTH, 2) uno spostamento a sinistra e in basso del set point della curva $\mathrm{Ca}-\mathrm{PTH}, 3)$ un miglioramento delle alterazioni istologiche (39, 40-44).

Occorre qui ricordare che il trattamento dell'iperparatiroidismo secondario severo con calcitriolo talora provoca una ipercalcemia persistente senza un adeguato controllo dei livelli di PTH. In questi casi una terapia prolungata può essere dannosa ed è quindi preferibile avviare il paziente ad un programma di paratiroidectomia. 
TABELLA VI - PARAMETRI BIOCHIMICI, CALCITRIOLO E DOSE DEI CHELANTI PRIMA E DOPO TRATTAMENTO CON CALCITRIOLO E.V.

\begin{tabular}{lcccc}
\hline & \multicolumn{2}{c}{$\mathrm{R}(\mathrm{n}=18)$} & \multicolumn{2}{c}{ NR $(\mathrm{n}=17)$} \\
\cline { 2 - 5 } & Prima & Dopo & Prima & Dopo \\
\hline Ca ionizzato $(\mathrm{mg} / \mathrm{dL})$ & $5.0 \pm 0.2$ & $5.2 \pm 0.2^{+}$ & $5.3 \pm 0.16$ & $5.4 \pm 0.1^{\#}$ \\
PTH $(\mathrm{pg} / \mathrm{mL})$ & $479 \pm 183$ & $175 \pm 63^{+}$ & $682 \pm 236$ & $703 \pm 180 \S$ \\
Fosforemia $(\mathrm{mg} / \mathrm{dL})$ & $4.7 \pm 0.8$ & $4.8 \pm 0.9$ & $5.0 \pm 0.7$ & $5.2 \pm 0.7$ \\
Fosfatasemia alcalina (UI/L) & $260 \pm 129$ & $179 \pm 82^{+}$ & $250 \pm 81$ & $210 \pm 78 \S$ \\
Dose calcitriolo $(\mu \mathrm{g} / \mathrm{sett})$ & 0 & $3.4 \pm 1.2$ & 0 & $4.0 \pm 1.6$ \\
Ca carbonato/acetato $(\mathrm{g} / \mathrm{die}) *$ & $1.9 \pm 1.2$ & $2.4 \pm 1.2$ & $1.7 \pm 1.1$ & $2.2 \pm 1.0$ \\
\hline
\end{tabular}

(*) come Ca elemento

$(+) p<0.001$ vs prima

(\#) $p<0.01$ vs $R$

(§) $p<0.001$ vs $R$
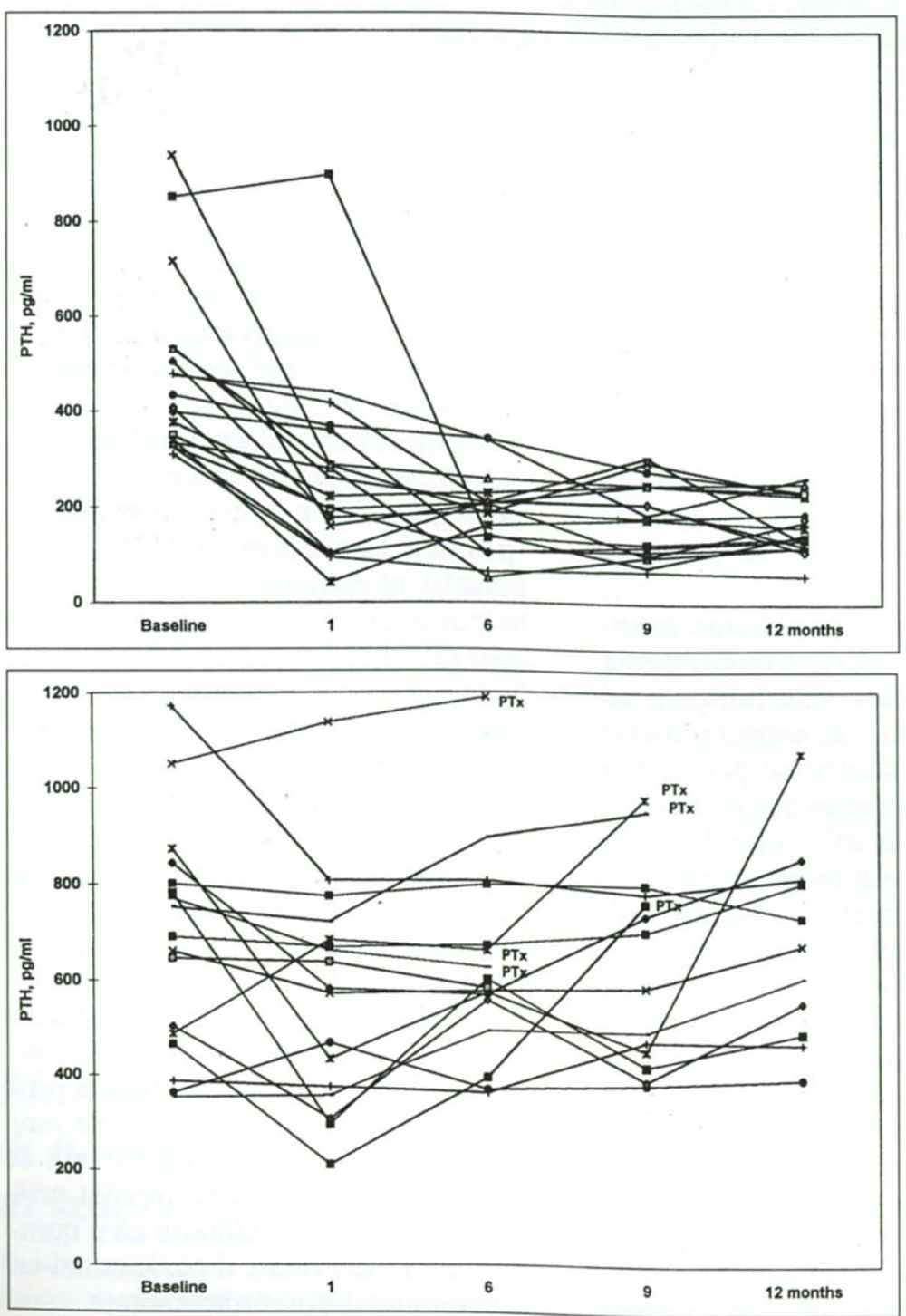

Fig. 1 - Effetti della terapia con calcitriolo e.v. sul paratormone intatto nei pazienti responsivi. (da Malberti et al (42)).

Fig. 2 - Effetti della terapia con calcitriolo e.v. sul paratormone intatto nei pazienti non responsivi. $\quad(d a$ Malberti et al (42)).

\section{Effetti a lungo termine del trattamento con calcitriolo}

Recentemente Malberti et al hanno condotto uno studio con lo scopo di valutare gli effetti del calcitriolo a boli e.v. sulla funzione paratiroidea ed i possibili fattori predittivi di una risposta negativa alla terapia (42).

Sono stati selezionati per il trattamento (Calcijex $2 \mu \mathrm{g}$ post-dialisi per 12 mesi) 35 pazienti con iperparatiroidismo secondario $(\mathrm{PTH}>325$ $\mathrm{pg} / \mathrm{mL}$ ). Prima dello studio è stata valutata la dimensione delle ghiandole paratiroidee (ecografia del collo con color-doppler) e alcuni parametri della relazione $\mathrm{Ca}-\mathrm{PTH}$ ottenuta inducendo ipocalcemia ed ipercalcemia così da stimolare o sopprimere in modo massimale la secrezione di PTH. Durante lo studio sono state somministrate dosi di calcio carbonato o calcio acetato adeguate a mantenere una fosforemia inferiore a $5.5 \mathrm{mg} / \mathrm{dL}$. In caso di ipercalcemia veniva ridotto il calcio nel dialisato fino a $5 \mathrm{mg} / \mathrm{dL}$ e, se necessario, anche la dose di calcitriolo. L'obbiettivo terapeutico era di ridurre il PTH sierico al di sotto di $260 \mathrm{pg} / \mathrm{mL}$ mantenendo la calcemia nel range di normalità; i pazienti che raggiungevano questo target venivano considerati responsivi. 
Considerando complessivamente tutti i 35 pazienti, alla fine del trattamento con calcitriolo abbiamo osservato una riduzione statisticamente significativa $(\mathrm{p}<0.01)$ della fosfatasemia alcalina e del PTH (in media del $30 \%$ ) e, al contrario, un aumento della calcemia ionizzata (Tab. VI).

I livelli di PTH si sono ridotti durante il periodo di terapia ed hanno raggiunto il target in 18 pazienti su 35 $(51.4 \%)$ che sono stati quindi definiti responsivi mentre i restanti 17 pazienti $(48.6 \%)$ non hanno raggiunto l'obbiettivo (non responsivi). Di questi, 14 pazienti hanno mostrato un PTH invariato o aumentato mentre 3 pazienti hanno avuto una riduzione del PTH fra il 20 e $35 \%$ con sviluppo di ipercalcemia.

La Figura I mostra le modificazioni assolute dei livelli di PTH nel corso del periodo di trattamento nei 18 pazienti responsivi. In questo gruppo i livelli di PTH si sono ridotti a partire dal primo mese mentre il target è stato raggiunto in 6 mesi in 16 pazienti su 18.

La Figura 2 mostra invece il trend dei livelli di PTH nel gruppo di pazienti non responsivi. Per 6 di essi si è resa necessaria la paratiroidectomia in seguito allo sviluppo di ipercalcemia persistente (nonostante la riduzione del calcio nel dialisato e delle dosi di calcitriolo fino al minimo previsto di I $\mu$ g per due volte la settimana) e progressione dei livelli di PTH.

Dato che la dose media di calcitriolo è risultata simile nei 2 gruppi in questo studio, la causa della refrattarietà al trattamento non può essere attribuita a questo fattore. Al contrario la capacità di ottenere un controllo a lungo termine dei livelli di PTH può essere stata limitata dalla necessità di ridurre le dosi a causa dell'ipercalcemia in alcuni singoli pazienti. Il ruolo negativo dell'iperfosforemia è stato eliminato sia per la selezione iniziale dei pazienti sia per lo stretto controllo durante il periodo di trattamento.

La Tabella VII mostra i parametri pre-trattamento dei 2 gruppi di pazienti responsivi e non come risultato del raggiungimento del target al termine dei 12 mesi di studio.

TABELLA VII - PARAMETRI DI FUNZIONE PARATIROIDEA ALL'INIZIO DELLO STUDIO

\begin{tabular}{|c|c|c|}
\hline & Responsivi $(\mathrm{n}=18)$ & Non responsivi $(\mathrm{n}=17)$ \\
\hline Calcio ionizzato (ICa), $\mathrm{mg} / \mathrm{dL}$ & $5.0 \pm 0.2$ & $5.3 \pm 0.16^{\#}$ \\
\hline PTH, pg/mL & $479 \pm 183$ & $682 \pm 236^{\S}$ \\
\hline $1 \mathrm{Ca} \max ^{1}, \mathrm{mg} / \mathrm{dL}$ & $4.36 \pm 0.16$ & $4.64 \pm 0.24^{\#}$ \\
\hline PTH max, pg/mL & $883 \pm 257$ & $1364 \pm 533^{\#}$ \\
\hline ICa $\min ^{2}, \mathrm{mg} / \mathrm{d} \mathrm{L}$ & $5.64 \pm 0.2$ & $5.92 \pm 0.24^{\#}$ \\
\hline PTH min, $\mathrm{pg} / \mathrm{mL}$ & $141 \pm 109$ & $327 \pm 110^{\S}$ \\
\hline PTH min/PTH max, \% & $16.0 \pm 6$ & $24.8 \pm 8^{\S}$ \\
\hline Set point ICa- $\mathrm{A}^{3}, \mathrm{mg} / \mathrm{dL}$ & $4.96 \pm 0.2$ & $5.32 \pm 0.2^{\#}$ \\
\hline Set point $1 \mathrm{Ca}-\mathrm{B}^{4}, \mathrm{mg} / \mathrm{dL}$ & $4.84 \pm 0.2$ & $5.16 \pm 0.16^{\#}$ \\
\hline Slope, $\% / \mathrm{mmol} / \mathrm{L}$ & $247 \pm 35$ & $237 \pm 44$ \\
\hline Volume paratiroidi, $\mathrm{cm}^{3}$ & $0.4 \pm 0.3$ & $2.4 \pm 2.1^{\#}$ \\
\hline \multicolumn{3}{|c|}{$\begin{array}{l}1 \text { ICa max = livello di calcemia ionizzata al quale si è avuta la massima secrezione di PTH } \\
2 \text { ICa min = livello di ICa al quale si è avuta la minima secrezione di PTH } \\
3 \text { set point ICa-A = livello di ICa al quale la massima secrezione di PTH è ridotta del } 50 \% \\
4 \text { set point ICa-B = livello di ICa corrispondente al punto intermedio tra PTH min e PTH } \\
\text { max sec. Brown et al. ( } 40 \text { ) } \\
\text { \# }=\mathrm{p}<0.001 ; \S=\mathrm{p}<0.01 \text { vs responsivi }\end{array}$} \\
\hline
\end{tabular}

I dati mostrano che la risposta è influenzata negativamente quando le ghiandole paratiroidee sono di grosse dimensioni (vol. $>1 \mathrm{~cm}^{3}$ ) e mostrano una ridotta sensibilità al calcio. Per quel che riguarda i parametri dinamici della relazione calcio-PTH abbiamo osservato che il PTH e la calcemia ionizzata basali, la massima secrezione di PTH indotta da ipocalcemia, la massima inibizione del PTH indotta da ipercalcemia, la calcemia ionizzata corrispondente alla massima secrezione e inibizione di PTH ed il set point del calcio erano significativamente più alti nei pazienti non responsivi. Questi dati nel loro insieme indicano che la curva calcio-PTH era spostata a $\mathrm{dx}$ in questi soggetti rispetto a quelli responsivi. L'analisi statistica con regressione logistica ha mostrato tuttavia che fra i parametri dinamici della funzione paratiroidea solo il set point ha un ruolo predittivo negativo (odds ratio, 0.0001; intervallo di confidenza 95\%).

Quindi, analogamente a quanto osservato da altri Autori $(44,45)$, si è confermata una relazione fra massa tumorale, anomalie funzionali e refrattarietà al calcitriolo delle cellule paratiroidee. La valutazione del volume del tessuto paratiroideo e del set point del calcio possono essere considerati indicatori utili per la decisione se protrarre il trattamento farmacologico o procedere alla ablazione chirurgica delle paratiroidi. In alternativa all'intervento può essere proposto un intervento di alcolizzazione, manovra meno invasiva, ma di minor efficacia e richiedente ripetute applicazioni. Inoltre in caso di insuccesso o successo parziale il successivo intervento chirurgico è reso più indaginoso e tecnicamente più difficile. 


\section{Conclusioni e prospettive}

Anche se in misura minore rispetto al passato, l'iperparatiroidismo rappresenta ancora una causa fortemente invalidante per alcuni pazienti uremici e comunque condizionante la loro qualità di vita. Il corretto trattamento e soprattutto una attenta politica di prevenzione costituiscono quindi una sfida sempre attuale per il nefrologo. Considerate le conoscenze che sull'argomento sono state acquisite o sono in via di acquisizione negli ultimi anni si conferma il fatto che una precoce restrizione dell'apporto fosforico dietetico e la supplementazione con metaboliti attivi della vitamina $\mathrm{D}$ costituiscono gli interventi cardine nel prevenire o nel controllare questa complicanza.

Per quanto concerne il controllo della fosforemia un particolare scrupolo è richiesto nell'evitare gel o prodotti contenenti alluminio anche in funzione gastroprotettiva. A questo proposito sembra promettente, ma ancora in uso sperimentale, l'impiego di chelanti del fosforo non tossici e privi di calcio. Per rimanere nel campo delle prospettive cliniche ormai prossime anche il trattamento farmacologico specifico dell'iperparatiroidismo sta acquisendo un numero crescente di informazioni su molecole recentemente introdotte come gli analoghi della vitamina $D$ non calcemizzanti ed i calcimimetici. È ancora prematuro per trarre conclusioni ma le esperienze fin qui riportate in letteratura consentono di guardare al futuro con speranza.

\section{BIBLIOGRAFIA}

1. Juppner H, Abou-Samra AB, Freeman MW, et al. Expression cloning of a common receptor for parathyroid hormone and parathyroid hormone-related peptide. Science 1991; 254: 1024-6.

2. Urena P, Segre GV, Drueke TB. Parathyroid hormone receptor. Nephrol Dial Transplant 1994; 9: 593-5.

3. Zerwekh JE. Bone metabolism. Seminars in Nephrology 1992; 12: 79-90.

4. Hruska Ka, Teitelbaum SL. Renal Osteodistrophy. N Engl J Med 1995; 333: 166-74.

5. Sutton RAL, Camerol EC. Renal Osteodystrophy. Patophysiology. Seminars in Nephrology 1992; 12: 91-100.

6. Chan YL, Furlong TJ, Cornish CJ, et al. Dialysis osteodystrophy. Medicine 1986; 64: 296-307.

7. Slatopolsky E, Delmez JA Pathogenesis of secondary hyperparathyroidism. Am J Kidney Dis 1994; 23: 229-36.

8. Drueke TB, Kubrusly M. Pathogenesis of secondary hyperparathyroidism in chronic renal failure. J Nephrol 1994; 7 : 139-47.

9. Silver J, Russell J, Sherwood LM. Regulation by Vitamin D metabolites of messenger ribonucleic acid for pre-proparathyroid hormone in isolated bovine parathyroid cells. Proc Natl Acad Sci, USA 1985; 82: 4270-3.

10. Brown AJ, Zhong M, Finch J, Ritter C, Slatopolsky E. The roles of calcium and 1,25 dihydroxyvitamin $\mathrm{D}_{3}$ in the regulation of vitamin $\mathrm{D}$ receptor expression by rat parathyroid glands. Endocrinology 1995; 136: 1419-5.

11. Kremer R, Bolivar I, Goltzman D, Hendy GN. Influence of calcium and 1,25 dihidroxycholecalciferol on proliferation and proto-oncogene expression in primary cultures of bovine parathyroid cells. Endocrinology $1989 ; 935-41$.

12. Tsukamoto Y. Pathophysiology and treatment of secondary hyperparathyroidism in patients with chronic renal failure. Nephrol Dial Transplant 1995; 10 (Suppl 3): 22-4.

13. Hsu CH, Patel SR, Young EN, Vanholder R. The biological action of calcitriol in renal failure. Kidney Int 1994; 46: 650-62.

14. Slatopolsky E, Caglar S, Pennell JP, Toggart J, Canterbury J, Reiss E, Bricker NS. On the pathogenesis of hyperparathyroidism in chronic experimental insufficiency in the dog. J Clin Invest 1971; 50: 497-9.

15. Falchetti A, Bale AE, Amorosi A, Bordi C, Cicchi P, Bandini S, Marx SJ, Brandi ML. Progression of uremic hyperparathyroidism involves allelic loss on chromosome 11. J Clin Endocrinol Metab 1993; 76: 139-44.

16. Llach F. Secondary hyperparathyroidism in renal failure: the trade-off hypothesis revisited. Am J Kidney Dis 1995; 25: 663-79.

17. Brown EM, Gamba G, Riccardi D, Lombardi D, Butters R, Kifor O, Sun A, Hediger MA, Litton J, Hebert SC. Cloning and characterization of an extracellular $\mathrm{Ca}^{2+}$ sensing receptor from bovine parathyroid. Nature 1993; 366: 575-80.

18. Goodman WG, Belin TR, Salusky JB. In vivo assessments of calcium-regulated parathyroid hormone release in secondary hyperparathyroidism. Kidney Int 1996; 50: 1834-44.

19. Felsenfeld AJ, Llach F. Parathyroid gland function in chronic renal failure. Kidney Int 1993; 43: 771-89.

20. Slatopolsky E, Delmez JA. Pathogenesis of secondary hyperparathyroidism. Nephrol Dial Transplant 1996; 11 (Suppl 3): 130-5.

21. Hebert SC. Extracellular calcium sensing receptor: implication 
for calcium and magnesum handling in the kidney. Kidney Int 1996; 50: 2129-39.

22. Rodriguez M, Felsenfeld AJ, Llach F. Calcemic response to parathyroid hormone in renal failure: role of calcitriol and the effect of parathyroidectomy. Kidney Int 1991; 40: 1063-8.

23. Rodriguez M, Martin-Malo A, Martinez ME, Torres A, Felsenfeld AJ, Llach F. Calcemic response to parathyroid hormone in renal failure: role of phosphorus and its effect on calcitriol. Kidney Int 1991; 40: 1055-62.

24. Yates AJ, Oreffo ROC, Mayor $\mathrm{K}$, Mundy GR. Inhibition of bone resorption by inorganic phosphate is mediated by both reduced osteoclasts formation and decreased activity of mature osteoclasts. J Bone Miner Res 1991; 6: 473-8.

25. Drueke TB. The pathogenesis of parathyroid gland hyperplasia in chronic renal failure. Kidney Int 1995; 48: 259-72.

26. Sherrard DJ, Oh S, Maloney N, Andress O, Coburn J. Renal osteodystrophy: classification, cause and treatment in "Frame B, Potts JJJr eds Clinical Disorders of bone and mineral metabolism". Excepta Medica, Amsterdam 1983, 254-9.

27. Gonzalez EA, Martin KJ: Renal osteodystrophy. Pathogenesis and management. Nephrol Dial Transplant 1995; 10 (Suppl 3): 13-21.

28. Wang M, Hercz G, Sherrard DJ Maloney NA, Segre GV, Pei Y. Relationship between intact 1-84 parathyroid hormone and bone histomorphometric parameters in dialysis patients without Aluminum toxicity. Am J Kidney Dis 1995; 26: 836-44.

29. Dunstan CR, Hills E, Norman AW, Bishop JE, Mayer E, Wong SYP, Johnson JR, George CRP, Collette P, Kalowski S, Wyndham R, Lawrence JR, Evans RA: The pathogenesis of renal osteodystrophy: Role of vitamin $\mathrm{D}$, aluminum, parathyroid hormone, calcium and phosphorus. Quart J Med 1985; 217: 127-44.

30. Malberti F, Surian M, Ionised calcium changes and parathyroid hormone secretion in hemodiafiltration in relation to substitution fluid content. Nephrol Dial Transplant 1991; 2: 104-7.

31. Malberti F, Surian M, Minetti L. Dialysate calcium concentration decrease exacerbates secondary hyperparathyroidism in dialysis patients given calcium carbonate as a phosphate binder. J Nephrol 1991; 2: 75-81.

32.

Fournier A, Morinier Ph, Marie A. Adynamic bone disease - Is it actually a disease? Nephrol Dial Transplant 1995; 10:454-7.

33. Goodman W, Ramirez J, Belin T, Chon Y, Gales B, Segre GV, Salusky IB. Development of adynamic bone in patients with secondary hyperparathyroidism after intermittent calcitriol therapy. Kidney Int 1994; 46: 1160-6.

34. Silver J, Naveh-Many T, Mayer H, Schmelzer HJ, Popovzter MN. Regulation by vitamin D metabolites of parathyroid hormone gene transcription in vivo in the rat. J Clin Invest 1986; 78: 1296-301.

35. Fukuda N, Tanaka H, Tominaga $\mathrm{Y}$, Fukagawa M, Korokawa $\mathrm{K}$, Seino Y. More severe form of parathyroid hyperplasia is associated with decreased calcitriol receptor density in chronic uremic patients. $\mathbf{J}$ Am Soc Neph 1992; 3: 695-9.

36. Kwan J TC, Almond ML, Beer JR, Noonan K, Evans SJW, Cunningham J: "Pulse" oral calcitriol in uraemic patients: rapid modification of parathyroid response to calcium. Nephrol Dial Transplant 1992; 7: 829-36.

37. Reichel H, Srabo A, Uhl J, Pesion S, Schmutz A, Schmidt-Gayk H, Ritz E. Intermittent versus continuous administration of 1.25 dihydroxyvitamin $\mathrm{D}$ in experimental renal hyperparathyrodism. Kidney Int 1993; 44: 1259-65.

38. Quarles LD, Yohay DA, Carroll $\mathrm{BA}$, et al. Prospective trial of pulse oral versus intravenous calcitriol treatment of hyperparathyroidism in ESRD. Kidney Int 1994; 45: 1710-21.

39. Slatopolsky E, Weerts C, Thie- lan J, Horst R, Harter H, Martin KJ. Marked suppression of secondary hyperparathyroidism by intravenous administration of 1.25 dihydroxycholecalciferol in uremic patients. $\mathbf{J}$ Clin Invest 1984; 74: 2136-43.

40. Gallieni M, Brancaccio D. Which is the preferred treatment of advanced hyperparathyroidism in a renal patient? I. Medical intervention is the primary option in the treatment of advanced hyperparathyroidism in chronic renal failure. Nephrol Dial Transplant 1994, 9: 1816-9.

41. Cannella G, Bonucci E, Rolla D, et al. Evidence of healing of secondary hyperparathyroidism in chronically hemodialysis uremic patients treated with long term intravenous calcitriol. Kidney Int 1994; 46: 1126-32.

42. Malberti F, Corradi B, Cosci P, Calliada F, Marcelli D, Imbasciati E. Long-term effects of intravenous calcitriol therapy on the control of secondary hyperparathyroidism. AJKD 1996; 28: 704-12.

43. Brown EM. Four-parameter model of the sigmoidal relationship between parathyroid hormone release and extracellular calcium concentration in normal and abnormal parathyroid tissue. J Clin Endocrinol Metab 1983; 56: 572-81.

44. Felsenfeld AJ, Jara A, Pahl M, Bover J, Rodriguez M. Differences in the dynamics of parathyroid hormone secretion in hemodyalisis patients with marked secondary hyperparathyroidism. J Am Soc Nephrol 1995; 6: 1371- 8.

45. Khosla S, Ebeling PR, Firek AF, Burritt MM, Kao PC, Heath H. Calcium infusion suggests a "set point" abnormality of parathyroid gland function in familial benign hypercalcernia and more complex disturbances in primary hyperparathyroidism. J Clin Endocrinol Metab 1993; 76: 715-20. 